\title{
IRAS, the human homologue of Nischarin, prolongs survival of transfected PC12 cells
}

\author{
Cell Death and Differentiation (2003) 10, 933-935. doi:10.1038/sj.cdd.4401275
}

\section{Dear Editor,}

IRAS was discovered only recently ${ }^{1}$ and is a highly unique protein with little structural similarity to known proteins listed in sequence databases. Nischarin was cloned as the mouse homologue of IRAS. ${ }^{2}$ The amino-acid sequence of Nischarin is about $80 \%$ homologous with IRAS, with a main difference being that Nischarin lacks the N-terminal 244 amino acids of IRAS. 1,2 Nischarin was reported ${ }^{2}$ to interact with the cytoplasmic tail of the integrin $\alpha 5$ subunit of the fibronectin receptor, and to inhibit cell migration and lamellipodia formation in transfected 3T3 cells. The human protein IRAS ( $h$ IRAS) was also found to interact with insulin receptor substrates (IRS) in HEK 293 cells and to enhance IRS4dependent insulin activation of extracellularly regulated kinase, ERK $1 / 2 .^{3}$ Herein, we report a new cellular role for IRAS, which is to allow PC12 cells to experience markedly less caspase-3 enzyme activity induced by apoptotic stimuli such as serum deprivation, thapsigargin, or staurosporine treatment. This decrease in apoptotic effector enzyme activity was accompanied by a decrease in the proportion of apoptotic cells presumably due to an increase in the delay to initiate apoptosis.

IRAS and Nischarin have been shown to exist in multiple tissues and cell lines. ${ }^{1-4}$ Rat IRAS is endogenously expressed in PC12 cells (Genbank EST 106159) as a $210 \mathrm{kDa}$ immunoreactive protein (Figure 1a), which is similar in size to the full-length form of Nischarin already detected in neuronal mouse cells. ${ }^{2}$ Three clonal sublines (IE10, 6E7, and 7D5) were selected by transfecting hIRAS cDNA into PC12 cells, isolating single cells, and propagating under geneticin selection. In addition, two empty-vector clonal sublines (pcDNA3.1 and 7D5a) were isolated in parallel by the same procedure. Cytologically, the cell lines were indistinguishable under phase contrast microscopy. Transfection of $h \mathrm{IRAS}$ into PC12 cells led to the appearance of the $167 \mathrm{kDa}$ human protein that was predominantly localized in the cytosol of cells (Figure 1a). The exact relation between the $210 \mathrm{kDa}$ rat IRAS (IRAS) and the $167 \mathrm{kDa}$ hIRAS protein is not fully known, since the rat homologue has only been partially sequenced (EST 106159). Based on rat and human tissue Northern blot analysis, ${ }^{4}$ it has been suggested that alternative splicing may be responsible for two molecular weight forms of this protein. If we assume that the two proteins play the same role in PC12 cells, then hIRAS-expressing PC12 cells constitute a moderate overexpression model of IRAS.

IRAS and Nischarin exhibit $100 \%$ amino-acid homology over an amino-acid sequence domain identified to bind $\alpha 5$ - integrin. ${ }^{2}$ The integrins, particularly the fibronectin receptor, have been linked to cell survival pathways. Overexpression of the $\alpha 5$ subunit protects cell lines against apoptotic stimuli, in part by modulating the expression of the antiapoptotic protein Bcl2 by activating the PI3kinase/Akt pathway. ${ }^{5,6}$ In this context, we hypothesized that IRAS might also modulate the survival of cells. An increase in caspase-3 activity is an index of apoptosis. We therefore measured the activities of caspase-3-like proteases after serum deprivation and compared the hIRAS-expressing and -nonexpressing cell lines. No differences in caspase-3 basal activities could be observed between the five cell lines grown in 15\% serumcontaining medium. However, by $3 \mathrm{~h}$ of serum starvation, caspase-3-like activities had increased dramatically in the control cells, attaining their plateau by $6 \mathrm{~h}$ (5.9-fold increase; Figure 1b). By comparison, caspase-3 activities in hIRAStransfected cells (i.e., IE10 clone) were increased four-fold by $6 \mathrm{~h}$ of serum starvation, but remained statistically less than those of control cells (Figure 1b) at least until $24 \mathrm{~h}$ in serumfree medium. As shown in Figure 1c, after 6 and $24 \mathrm{~h}$ of serum deprivation, higher levels of enzymatic activity were found in both control cell lines (pcDNA3.1 and 7D5a) compared to all hIRAS-expressing cell lines (IE10, 7D5 and 6E7 lines). Assay of Annexin V-FITC binding followed by flow cytometric measurements was next performed to evaluate quantitatively the apoptotic population in hIRAS-expressing and -nonexpressing cell lines. As shown in Figure 1d, about $60 \%$ of control cells (pcDNA3.1 and 7D5a clones) appeared apoptotic (Annexin V-FITC positive, lower right quadrant) after $48 \mathrm{~h}$ in serum-free medium. Under the same conditions, hIRASexpressing sublines (IE10, 7D5, and 6E7) showed a marked reduction in apoptotic populations (Figure 1d). Thus, reduced caspase-3 activation in serum-deprived hIRAS-expressing cells seemed to correlate with a reduction in the apoptotic cell population. Next, cell growth rate and survival in serumcontaining medium or in serum-free medium, respectively, were assessed microscopically. In this set of experiments, the number of viable cells were recorded by counting trypan blue excluding cells with a hemocytometer after different periods of time. The growth curves indicated that hIRAS-expressing cells behave similarly as control cells in serum-containing medium (data not shown). However, serum deprivation resulted in the death of $45 \%$ control cells within $24 \mathrm{~h}$ versus that of $0 \%$ hIRAS-expressing IE10 cells (Figure 1e). After $72 \mathrm{~h}$ serum deprivation, all the hIRAS-expressing clones (IE10, 7D5, and 6E7) showed a greater proportion of viable cells as assessed by trypan blue exclusion than control cell lines (pcDNA3.1 and 7D5a) (data not shown). Thus, another main 
a

+EmptyVector Wild Type +hIRAS

Memb Cyt Memb Cyt Memb Cyt

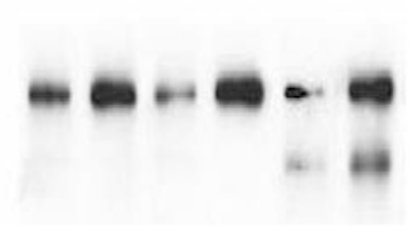

b

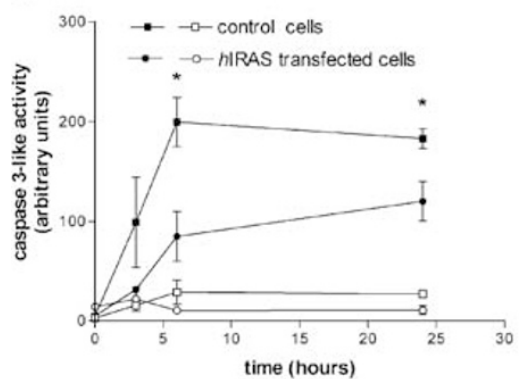

C

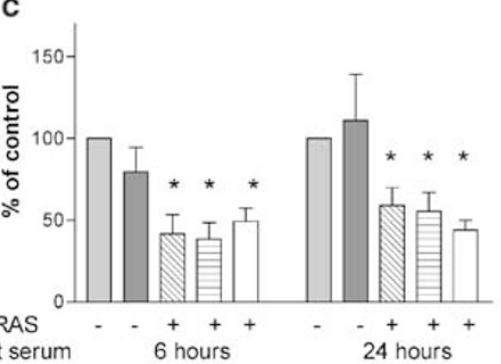

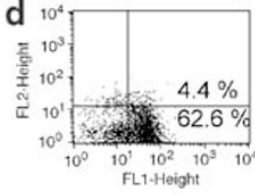

control cells

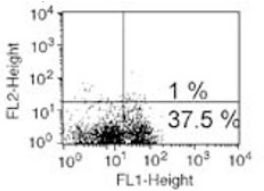

hIRAS expressing cells

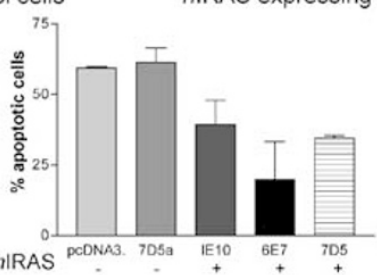

e
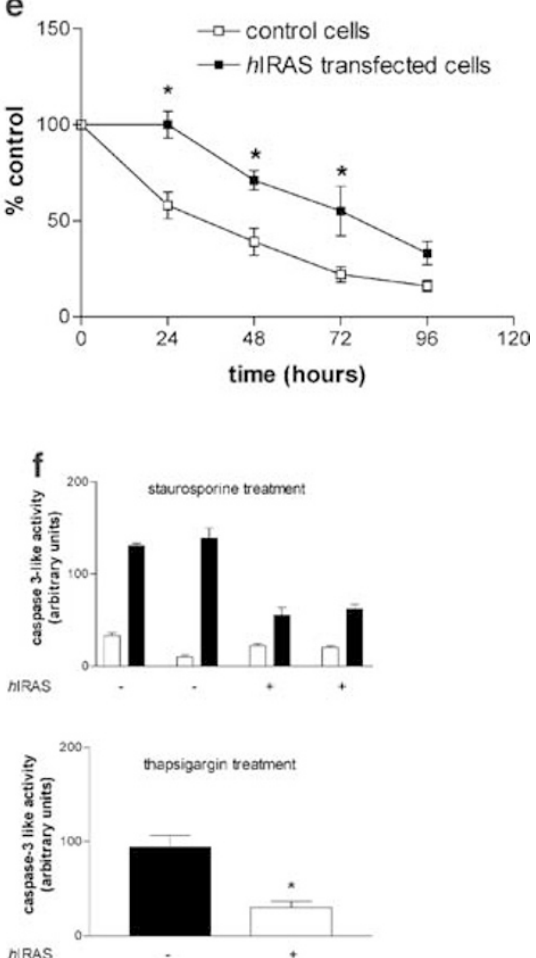

Figure 1 (a) Western blotting of $h$ IRAS-transfected PC12 cell membrane and cytosolic fractions. Immunorevelation was performed using 1201 antiserum specific for human IRAS ${ }^{1}$ with enhanced chemiluminescence. Memb, membranes; cyt, cytosol. (b) Effect of $h$ IRAS transfection on caspase-3 activity in serum-deprived cells. PC12 cells transfected with empty vector (pcDNA3.1) and hIRAS-transfected cells (IE10 subclone) were grown in complete (open symbols) or serum-free (closed symbols) medium. At the indicated times, cell lysates were prepared and used to determine caspase enzymatic activities by measuring the release of the para-nitroaniline chromophore from peptide substrate (DEVD) selective for caspase-3-like protease. Background levels were determined by parallel incubations of samples in the presence of caspase-3-like inhibitor, Ac-DEVD-CHO $(10 \mu \mathrm{M})$ and subtracted for each individual value. Enzymatic activity is expressed in arbitrary units as $0 . \mathrm{D} / \mu \mathrm{g}$ protein for each sample. Data are the mean \pm S.E.M. of triplicate determinations from three experiments. ${ }^{*} P<0.05$ compared to $h \mathrm{RAS}$-transfected cells. (c) PC12 cells either expressing $h$ IRAS (IE10, 7D5, and 6E7 sublines) or non-expressing hIRAS (pcDNA3.1 and 7D5a sublines) were grown in serum-free medium for 6 and $24 \mathrm{~h}$. Caspase-3 activities were measured as in (b) Results are expressed as percent activity recorded in pcDNA3.1 cells for each experiment. Mean \pm S.E.M. of three to five independent experiments. ${ }^{*} P<0.03$ as compared to pcDNA3.1 cell line. (d) hIRAS-mediated protection of apoptosis measured by Annexin V-FITC staining and flow cytometry. Upper two panels, control cells or hIRAS-expressing cells were grown for $48 \mathrm{~h}$ in serum-free medium before harvesting and analysis. Percentage values represent late apoptosis (upper right quadrant) and early apoptosis (lower right quadrant) populations of cells. In each case, a representative experiment out of three is shown. Lower panel: results are mean \pm S.E.M. of three independent experiments. The late and early apoptotic cells were taken together for this purpose. (e) Survival curves of empty vector and $h$ IRAS-transfected cells. PC12 cells stably transfected with empty vector (pcDNA3.1 subclone) or $h \mathrm{IRAS}$ (IE10 subclone) were recovered after 24, 48, 72, and $96 \mathrm{~h}$ in serum-free medium and viable cells excluding trypan blue were counted. Results are expressed as percent of control at time $0 \mathrm{~h}$ for each cell line. Data shown are mean \pm S.E.M. of triplicate determinations from three to six independent experiments. ${ }^{\star} P<0.05$ as compared to pcDNA3.1 cells. (f) Upper graph: Effect of $h \mathrm{RAS}$ transfection on staurosporine-induced caspase-3 activation. PC12 cells not expressing hIRAS (pcDNA3.1 and 7D5a sublines) or expressing hIRAS (IE10 and 7D5 sublines) were grown in complete medium (15\% serum) in the absence (open bars) or presence (closed bars) of staurosporine ( $1 \mu \mathrm{M})$ for $6 \mathrm{~h}$. Results are expressed as the mean \pm S.E.M. of three independent experiments each performed in triplicate. Lower graph: Effect of $h \mathrm{RAS}$ transfection on thapsigargin-induced caspase-3 activation. PC12 cells transfected with empty vector (pcDNA3.1 subline) and IRAS-transfected cells (IE10 subline) were grown in $5 \%$ serum-containing medium with thapsigargin $(100 \mathrm{nM})$ for $6 \mathrm{~h}$. Results are expressed as the mean \pm S.E.M. of two independent experiments each performed in sextuplicate. ${ }^{*} P<0.01$ as compared to pcDNA3.1 cells. 
difference between control cells and hIRAS-expressing cell lines is prolonged survival of the latter in serum-free conditions, which may be related to the decrease in caspase-3 activity and apoptotic cell population observed previously. When serum is readded after $24 \mathrm{~h}$ serum deprivation, we observed that $h \mathrm{IRAS}$-expressing cell lines proved able to proliferate again in marked contrast with control cells that continued to die $(56 \pm 15 \%$ and $250 \pm 67 \%$ of trypan blue excluding cells after $72 \mathrm{~h}$ in serum-containing medium for control and $h$ IRAS-expressing cells, respectively; $100 \%$ refers to the viable cells recorded after $24 \mathrm{~h}$ serum deprivation in each cell line).

In an attempt to generalize that transfection of $h \mathrm{IRAS}$ delays PC12 cells apoptosis, treatments were next performed with two different cytotoxic agents, thapsigargin and staurosporine. Thapsigargin, a $\mathrm{Ca}^{2+}$-ATPase inhibitor that increases intracellular calcium by inhibiting the uptake of calcium into the endoplasmic reticulum, is known to induce apoptosis in many cell types including PC12 cells. ${ }^{7}$ Staurosporine, a protein kinase inhibitor, also induces apoptosis in PC12 cells. ${ }^{8}$ Staurosporine treatment of PC12 cells induces a sustained elevation of intracellular calcium and accumulation of reactive oxygen species. ${ }^{8}$ Both of these apoptotic stimuli are known to trigger caspase-3 activation. ${ }^{7,8}$. Thapsigargin was applied to the cells in $5 \%$ serum-containing medium, and then caspase-3 activities were recorded. As shown in Figure 1f, thapsigargin (100 nM) enhanced caspase-3 activity in control cells (pcDNA3.1 clone). In contrast, no enhancement by thapsigargin of caspase-3 activity was observed in hIRAS-expressing cells (IE10 clone). Staurosporine $(1 \mu \mathrm{M})$ was added to complete medium (containing $15 \%$ serum), thus excluding any impact of low serum concentration on caspase3 activity. In this paradigm, the sublines stably expressing hIRAS (1E10 and 7D5) showed lower caspase-3 activation after $6 \mathrm{~h}$ treatment with $1 \mu \mathrm{M}$ staurosporine compared to control cells (pcDNA3.1 and 7D5a clones) (Figure 1f). Caspase-3 activity after $6 \mathrm{~h}$ was increased 3.6- and 8.8-fold in control sublines (pcDNA3.1 and 7D5a, respectively) versus 2.3- and 2.8-fold in hIRAS-expressing cells (IE10 and 7D5 clones respectively). We thus have confirmed the activation of this apoptosis effector enzyme by these two drugs, and in the process shown that hIRAS also inhibits the increase in caspase-3 activity in these conditions.

In summation, IRAS appears to impinge on a mechanism central to apoptosis induced by distinct stimuli and which lies upstream of the caspase- 3 activation resulting at least in a delayed cell death. According to the results of Figure 1e, it seems that a continued proapoptotic stimulus ( $96 \mathrm{~h}$ serum deprivation, for example) eventually overrides the IRAS block of apoptosis. As readdition of serum at an earlier time point of apoptosis triggering (after $24 \mathrm{~h}$ serum deprivation) allowed the hIRAS-expressing cells to divide again, we speculate that $h$ IRAS lowers the probability of initiation of the apoptotic program rather than prolonging each individual apoptotic event as described for caspase-3 inhibitors. ${ }^{9}$ Thus, hIRAS blocks apoptosis before destruction of membranes and loss of phosphatidylserine asymmetry and seems to protect the cells from the loss of the proliferative potential. Intriguingly, it appears from the literature that expression of the $\alpha 5$ integrin subunit delays serum-deprivation-induced as well as staur- osporine-induced apoptosis in a manner very similar to the one obtained by the transfection of $h$ IRAS. ${ }^{10}$

The mechanism of action of IRAS remains unresolved at this time and is currently under investigation. Hypotheses have been tested according to the proteins that are known to interact with IRAS. ${ }^{2,3}$ IRAS may be implicated either in the insulin or the integrin dependent survival pathway, both of which imply PI3-kinase activation. ${ }^{11,6}$ However, our assays to inhibit the protective effect of IRAS with LY294002 or wortmannin (two PI3-kinase inhibitors) were unsuccessful; although a little enhancement of caspase-3 activity in serumdeprived $h \mathrm{R}$ RAS-expressing clones was recorded, no significant increase in the proportion of apoptotic cells could be reached with these inhibitors (data not shown). Likewise, PD98059, an inhibitor of the ERK1/2 pathway, was unable to induce an increase in hIRAS-expressing apoptotic cells (data not shown).

Our study has thus identified a new cellular role for the recently cloned human protein IRAS. We demonstrated that hIRAS expression in PC12 cells results in protection against apoptosis over a 2-3-day period of time. Although the mechanism of action of IRAS is not resolved, it may represent a previously unknown class of protein modulating the fine regulation of cell death and growth.

\section{Acknowledgements}

We thank Dr. Peter Eickelman (Solvay Pharmaceuticals, Hannover, Germany) for advice with the transfection protocol, and Mary Elise Lutrick (University of Mississippi Medical Center) for carefully selecting and maintaining the sublines. This work was partially supported by a grant from Solvay Pharma (Hanover, Germany) and Grant MH49248 from the National Institute of Mental Health (USA) for J Piletz's group.

$M$ Dontenwill $^{1 *}$, G Pascal, JE Piletz ${ }^{3}, M$ Chen $^{3}$, J Baldwin ${ }^{3}, P$ Rondé $^{1}, L$ Dupuy $2, D$ Urosevic ${ }^{2}$, H Greney ${ }^{2}$, K Takeda $^{1}$ and $P$ Bousquet ${ }^{2}$

${ }^{1}$ Pharmacologie et Physicochimie des Interactions Cellulaires et Moléculaires, UMR 7034 CNRS, Faculté de Pharmacie, Université Louis Pasteur de Strasbourg, Illkirch, France

${ }^{2}$ Laboratoire de Neurobiologie et Pharmacologie Cardiovasculaire, Faculté de Medecine, Strasbourg, France

${ }^{3}$ Department of Psychiatry, Division of Neurobiology and Behavior Research, University of Mississippi Medical Center, Jackson, MS, USA

* Corresponding author: M Dontenwill, Pharmacologie et Physicochimie des Interactions Cellulaires et Moléculaires, UMR CNRS 7034, Faculté de Pharmacie, Université Louis Pasteur de Strasbourg, Illkirch, France. Tel: + 33 3 90244267; Fax: + 333 90244313; E-mail: mdontenwill@ aspirine.ustrasbg.fr

1. Piletz et al. (2000). DNA Cell Biol 19: 319-329

2. Alahari et al. (2000). J. Cell. Biol. 151: 1141-1154

3. Sano et al. (2002). J. Biol. Chem. 277: 19439-19447

4. Ivanov et al. (1998). J. Auton. Nerv. Sys. 7: 98-110

5. Matter and Ruoslahti (2001). J Biol. Chem. 276: 27757-27763

6. Lee and Juliano (2000). Mol. Biol. Cell 11: 1973-1987

7. Takadera and Ohyashiki (1998). Biochim. Biophys. Acta 1401: 63-71

8. Kruman et al. (1998). J. Neurosci. Res. 51: 293-308

9. McCarthy et al. (1997). J. Cell. Biol. 136: 215-227

10. O'Brien et al. (1996). Exp. Cell. Res. 224: 208-213

11. Barber et al. (2001). J. Biol. Chem. 276: 32814-32821 